\title{
Agregación coloidal en presencia de albúmina de suero bovino: influencia del grado de recubrimiento
}

\author{
M. TIRADO-MIRANDA ${ }^{1}$, A. SCHMITT' ${ }^{1}$, J. CALLEJAS-FERNÁNDEZ ${ }^{1}$, A. FERNÁNDEZ-BARBERO ${ }^{2}$ \\ ${ }^{1}$ Grupo de Física de Fluidos y Biocoloides, Departamento de Física Aplicada, Universidad de Granada, 18071 Granada, España. \\ ${ }^{2}$ Grupo de Física de Fluidos Complejos, Departamento de Física Aplicada, Universidad de Almería, 04120 Almería, España.
}

\begin{abstract}
En este trabajo, se presenta un estudio sobre la contribución de los diferentes mecanismos de agregación de partículas coloidales modificadas superficialmente. Las características de las partículas se modificaron adsorbiendo diferentes cantidades de Albúmina de Suero Bovino (BSA). Las propiedades electroquímicas del medio se fijaron a concentraciones de electrolito cercanas y superiores a la c.c.c. y a un $\mathrm{pH}$ muy cercano al punto isoeléctrico de la proteína. La dispersión estática y dinámica de luz se utilizaron para determinar la dimensión fractal de los agregados, $d_{f}$ así como la velocidad de agregación, $k_{s^{\prime}}$ en función del grado de recubrimiento. Los resultados experimentales muestran que la proteína es responsable de una reorganización estructural interna dentro de los agregados. Se encontró estabilidad estérica para altos grados de recubrimiento. Sin embargo, no se detectó floculación débil, convirtiéndose la coagulación y la floculación por puenteo en los mecanismos de agregación predominantes.
\end{abstract}

Palabras clave: agregación coloidal, adsorción de proteinas, dimensión fractal, dispersión estática y dinámica de luz

\section{Colloidal Aggregation in presence of Bovine Serum Albumin: influence of the degree of coverage}

In this paper, a study on the contribution of the different aggregation mechanisms of surface modified colloidal particles is presented. The particle surface characteristics were modified by adsorbing different amounts of Bovine Serum Albumin (BSA). Ion concentration was set close and higher than to the critical coagulation concentration (c.c.c.) and the working $\mathrm{pH}$ was adapted to the isoelectric point for the protein. Static and dynamic light scattering were employed to determine the cluster fractal dimension, $d_{f}$, and the rate of aggregation, $k_{s}$, as a function of the degree of coverage. Experimental results show structural rearrangement within the clusters due to the presence of protein on the particle surface. Steric stability was observed for the highest BSA degree of coverage. Weak flocculation was not present and coagulation and bridging flocculation mechanisms appear to be as clear candidates for explaining the experimental results.

Key words: Colloidal aggregation, protein adsorption, fractal dimension, static and dynamic light scattering

\section{INTRODUCCIÓN}

Es bien conocido que las macromoléculas que se adsorben de forma irreversible en la superficie de las partículas coloidales afectan fuertemente la estabilidad de los coloides (1, 2). A altos recubrimientos superficiales, los efectos estéricos impiden la floculación y conducen a un incremento en la estabilidad, mientras que a bajos recubrimientos, se forman puentes entre las macromoléculas que llevan a la floculación por puenteo [1,2]. Este mecanismo de agregación ocurre cuando una parte recubierta de la superficie de una partícula colisiona con una parte sin recubrir de otra partícula. En este caso, la velocidad de agregación depende fuertemente del grado de recubrimiento superficial.

Una forma de estudiar el mecanismo de agregación es mediante la velocidad de agregación. Son varios los modelos teóricos desarrollados para explicar la dependencia de esta velocidad con el grado de recubrimiento superficial. Uno de los modelos más simples es el propuesto por Healy y La Mer (3) quienes asumen que la colisión de las partículas está controlada únicamente por la difusión de las mismas y que la probabilidad de unión entre ellas es la unidad. Estos autores proponen que la velocidad de floculación por puenteo pura, $k_{s}$, es proporcional al número de sitios libres de proteína en una partícula, $\theta$, y al número de sitios ocupados en la otra, (1- $\theta)$, y viene dada por: $\mathrm{k}_{\mathrm{s}} \propto \theta(1-\theta)$. Este modelo implica que el máximo de floculación por puenteo ocurre a recubrimientos intermedios y no existe floculación ni entre partículas totalmente recubiertas ni entre partículas sin recubrir. Además, ellos no consideran otros mecanismos de agregación, lo que significa que la formación de agregados ocurre únicamente por puenteo.

Modelos posteriores a éste fueron desarrollados por Hogg (4), Ash and Clayfield (5), Moudgil (6) and Molski (7). Todos ellos hacen una extensión del modelo de Healy y La Mer. Aunque consideran nuevos mecanismos de agregación mantienen la floculación por puenteo como un mecanismo totalmente efectivo a recubrimientos intermedios, independientemente de las condiciones experimentales. Schmitt y cols. [8] proponen un modelo extendido que considera una probabilidad de colisión para cada mecanismo de agregación.

El objetivo de este trabajo es separar y cuantificar la contribución de los diferentes mecanismos de agregación que se manifiestan en un proceso de agregación coloidal. Mostraremos como la floculación por puenteo puede estar presente aunque no se observe un máximo en la dependencia de la velocidad de agregación con el grado de recubrimiento, y como las diferentes propiedades electroquímicas del medio de suspensión afectan a la contribución de los diferentes mecanismos de agregación. 


\section{TEORÍA}

\section{Dependencia de la velocidad inicial de agregación con el grado de cobertura superficial}

La cinética de los procesos de agregación en suspensiones coloidales puede ser descrita mediante la evolución temporal de la distribución en tamaño de los agregados, $\mathrm{Nn}(\mathrm{t})$. Para sistemas coloidales diluidos, donde únicamente se tienen en cuenta colisiones binarias, von Smoluchowski propuso el siguiente sistema de ecuaciones (9):

$$
\frac{d N_{n}}{d t}=\frac{1}{2} \sum_{i+j=n} k_{i j} N_{i} N_{j}-N_{n} \sum_{k=1}^{\infty} k_{n k} N_{k}
$$

Los parámetros $k_{i j}$ representan la velocidad a la cual un $i$-mero se enlaza con un $j$-mero. Ellos se interpretan en términos de una probabilidad de choque para dos agregados que difunden uno hacia otro y contienen toda la información física del sistema. En la aproximación de Smoluchowski's, todas las colisiones entre dos partículas son efectivas y la agregación está enteramente controlada por la difusión-browniana. Este es el modo de agregación más rápido posible si no se consideran otros mecanismos de transporte adicionales $(10,11)$.

La probabilidad de unión entre dos partículas coloidales, depende no solo de las fuerzas que actúan entre las partículas sino también, de que la parte de superficie que colisiona esté recubierta de macromoléculas o no. Teniendo esto en cuenta, se distinguen tres posibles mecanismos de agregación:

i. Coagulación. Se produce cuando colisionan dos partes no recubiertas. Este proceso corresponde a la agregación de dos partículas desnudas. La velocidad de reacción para esta configuración viene dada por $k_{c}$.

ii. Floculación débil. En este caso se produce la colisión de dos partes recubiertas. Este mecanismo de agregación está caracterizado por la velocidad de reacción $k_{f d}$.

iii. Floculación por puenteo. Colisión entre una parte sin recubrir y otra recubierta. En esta configuración las macromoléculas pueden formar puentes entre las partículas. Esta reacción vendrá caracterizada por $k_{f p}$

Teniendo en cuenta los mecanismos anteriores, la velocidad total de agregación será suma de todas y cada una de las aportaciones:

$$
k_{s}=k_{c}(1-\theta)^{2}+k_{f d} \theta^{2}+2 k_{f p} \theta(1-\theta)
$$

donde el factor 2 considera la probabilidad de choque entre una parte no recubierta de una partícula con una parte cubierta de otra partícula y viceversa. La ecuación [2] permite cuantificar la contribución de los diferentes mecanismos de agregación si se conoce la velocidad de reacción como función del grado de recubrimiento.

\section{Determinación experimental de la velocidad de reacción}

La función de autocorrelación de la intensidad dispersada se obtiene a partir del producto de la intensidad dispersada en un tiempo $t$ y un tiempo $t+\tau$ más tarde, $G(\tau)=\langle I(t) I(t+\tau)>$. Ésta se relaciona con la función de autocorrelación del campo dispersado, $g^{1}(\tau)$, mediante la relación de Siegert:

$$
g^{2}(\tau)=1+C\left|g^{1}(\tau)\right|^{2}
$$

donde $C$ es una constante que depende de la óptica del instrumento y $g^{2}(\tau)$ es la función de autocorrelación de la intensidad normalizada, que viene dada por (12):

$$
g^{2}(\tau)=\frac{\langle I(t) I(t+\tau)>}{<I(t) I(t)>}
$$

La información sobre la distribución en tamaño de los agregados se obtiene de los coeficientes del desarrollo en serie del logaritmo de la función de autocorrelación de campo (13):

$$
\ln g^{1}(\tau)=-\mu_{1} \tau+\mu_{2}\left(\frac{\tau^{2}}{2}\right)+\mu_{3}\left(\frac{\tau^{3}}{3 !}\right)+\ldots
$$

Este es el conocido método de los cumulantes y es ampliamente utilizado para el análisis de los datos de dispersión dinámica de luz. El primer cumulante, $\mu_{1}$, se relaciona con el coeficiente de difusión medio de las partículas, $\langle D\rangle$, mediante $\mu_{1}=<D>q^{2}$. Conocido el coeficiente de difusión medio, se calcula el radio hidrodinámico mediante la ecuación de Einstein-Stokes (12).

Por otra parte, considerando la solución de escalado dinámico de la ecuación de Smoluchowski y el momento $\alpha$-ésimo de la distribución de tamaños, Olivier y Sorensen (14) obtuvieron la siguiente ecuación para el primer cumulante de la función de autocorrelación de la intensidad dispersada:

$$
-\mu_{1}(t)=\mu_{1}(0)\left(1+\frac{t}{t_{c}}\right)^{-1 /(1-\lambda)} d f
$$

donde $t_{c}=2 / N_{0} k_{s}$ es el tiempo característico de agregación que depende de la concentración inicial de partículas, $N_{0}, y$ de la constante de velocidad para la formación de dímeros, $t_{c}$ De la ecuación (6) se obtiene el tiempo característico de agregación, $t_{c}$, si la dimensión fractal de los agregados, $d_{f}$ y el parámetro de homogeneidad de van Dongen y Ernst, $\lambda$, se conocen.

La dimensión fractal de los agregados se determinó por dispersión estática de luz a partir de la dependencia de la intensidad dispersada con el vector de dispersión. Mientras que el parámetro $\lambda$ se obtuvo del comportamiento asintótico de la evolución temporal del tamaño medio de los agregados, medido por dispersión dinámica de luz. Para más detalles ver (15).

\section{PROCEDIMIENTO EXPERIMENTAL}

Una suspensión acuosa de partículas monodispersas de poliestireno se eligió como sistema coloidal modelo. El radio de las partículas era de $(50 \pm 1) \mathrm{nm}$, con un índice de polidispersidad de $(0.09 \pm 0.02)$. El látex se sintetizó en presencia de un surfactante (MA80), cuyas moléculas se adhieren a la superficie de las microesferas de látex y consiguen estabilizar el sistema. La carga negativa de las partículas proviene de los grupos sulfato superficiales. La correspondiente densidad superficial de carga (-2.7 \pm 0.1$)$ $\mu \mathrm{C} / \mathrm{cm}^{2}$, se determinó por valoraciones conductimétricas y potenciométricas. La estabilidad se estimó determinando la concentración crítica de coagulación (c.c.c.) a partir de la evolución temporal de la intensidad dispersada. El valor obtenido para la c.c.c. fue $(0.495 \pm 0.007) \mathrm{M}$.

Como macromolécula modelo a adsorber en la superficie de las partículas se eligió la Albúmina de Suero Bovino (BSA) 
comercial. El único tratamiento previo antes de la adsorción fue la limpieza por diálisis. Diferentes cantidades de BSA se añadieron a una cantidad fija de suspensión coloidal tamponada. El pH de la suspensión se fijó cerca del punto isoeléctrico de la BSA, concretamente en $\mathrm{pH} 4.8$, para facilitar la adsorción de la proteína en la superficie de las partículas. Tras un periodo de incubación, las muestras se centrifugaron y la cantidad de proteína no adsorbida se midió espectrofotométricamente. La isoterma de adsorción (Figura 1) muestra la alta afinidad de la BSA por la superficie de las partículas de látex. Ésta se adsorbe hasta alcanzar un plateau final que indica la adsorción de una monocapa completa de proteína. Este método da la posibilidad de obtener partículas con un grado de recubrimiento superficial bien conocido controlando, simplemente, la cantidad de proteína añadida. Las partículas cubiertas fueron redispersadas y almacenadas a $\mathrm{pH} 7$ para evitar la agregación espontánea de los complejos látex-proteína.

Antes de realizar las agregaciones, las muestras fueron sometidas a ultrasonidos durante $15 \mathrm{~min}$ para asegurar condiciones iniciales monodispersas. La agregación fue inducida añadiendo un electrolito simple $\mathrm{KCl}$ a una muestra estable con un grado de recubrimiento bien conocido. La mezcla se realizó inyectando en la célula de medida, con un dispositivo en forma de $\mathrm{Y}$, iguales cantidades de complejos látex-proteína y de electrolito tamponado. Se estudiaron dos concentraciones de electrolito, una correspondiente a la c.c.c. del látex sin proteína adsorbida $(0.495 \mathrm{M})$ y otra superior a la c.c.c. (0700M). El pH para la agregación se fijó en pH4.8 mediante un tampón acético de baja fuerza iónica. La temperatura de trabajo se mantuvo constante en $(25 \pm 1)^{\circ} \mathrm{C}$.

Los experimentos de agregación se estudiaron mediante un dispositivo óptico de dispersión de luz que permite trabajar con dos técnicas simultáneamente: dispersión dinámica de luz y dispersión estática de luz (16). La primera mide la función de autocorrelación de la intensidad dispersada en diferentes tiempos durante el proceso de agregación y para un ángulo de dispersión de $60^{\circ}$. El análisis de los datos se realizó con nuestros propios programas informáticos. De esta forma se obtuvo el número medio de partículas por agregado en función del tiempo (15). La dispersión estática de luz mide la intensidad media dispersada para diferentes ángulos de dispersión. En nuestros experimentos el intervalo angular empleado fue de $10^{\circ}$ a $150^{\circ}$. Cuando la estructura final de los agregados está totalmente establecida, la intensidad media $I(q)$ muestra un comportamiento asintótico como se predice teóricamente. La dimensión fractal se determinó de este comportamiento asintótico de la intensidad dispersada, $I(q) \propto q^{\text {ddf }}$, para tiempos largos, válida en el intervalo $\left\langle R_{h}>^{-1} \leq q \leq<R_{0}>^{-1}\right.$, donde $q=(2 \pi n / \lambda) \operatorname{sen}(\theta / 2)$ es el vector de dispersión [16]. En la última expresión, $n$ es el índice de refracción del medio, $\lambda$ es la longitud de onda de la luz incidente y $\theta$ es el ángulo de dispersión.

\section{RESULTADOS Y DISCUSIÓN}

La figura 2 muestra los valores de la dimensión fractal en función del grado de recubrimiento de las partículas coloidales. Las agregaciones se realizaron a $\mathrm{pH} 4.8$ y a dos concentraciones de electrolito $\mathrm{KCl}$. En ambos casos se observa como la dimensión fractal de los agregados aumenta con el grado de recubrimiento, obteniéndose estructuras cada vez más compactas. Esto significa que la distribución espacial de masa en el interior de los agregados depende de las características superficiales de los mismos, siendo las moléculas de proteína

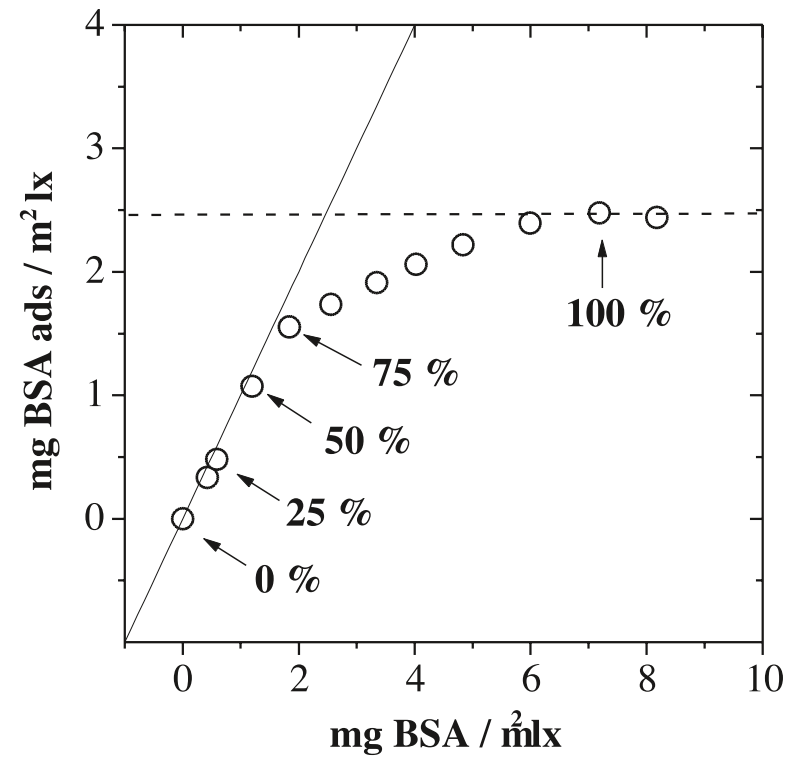

Figura 1: Isoterma de adsorción con BSA como macromolécula adsorbida. La adsorción se realizó a pH4.9.

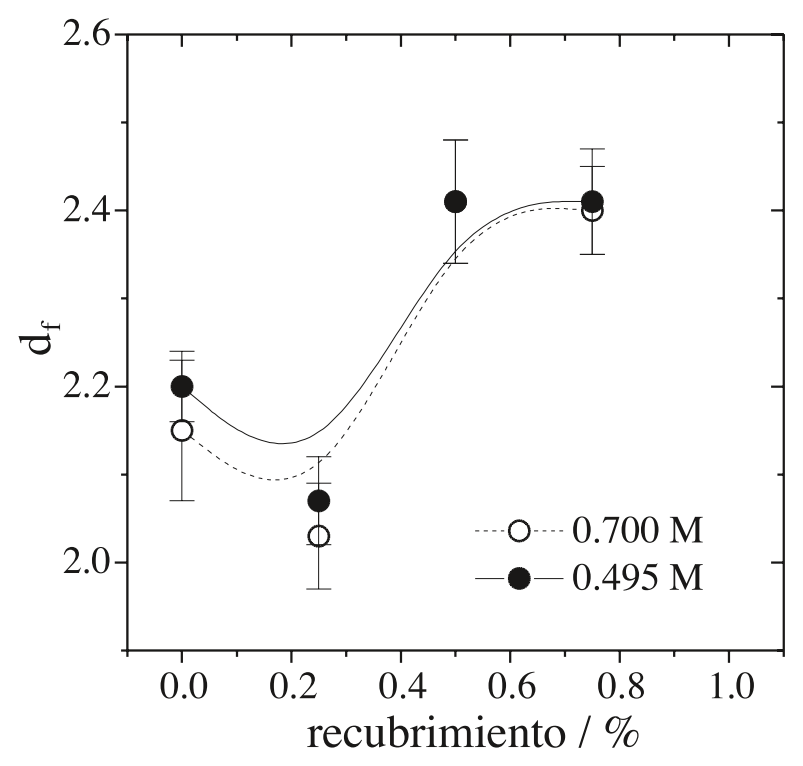

Figura 2: Dependencia de la dimensión fractal de los agregados recubiertos con proteína $(0 \%, 25 \%, 50 \%, 75 \%)$ para dos concentraciones de electrolito, $0.495 \mathrm{M}$ y $0.700 \mathrm{M}$.

adsorbidas las responsables de un proceso de reestructuración dentro del agregado (17). Cuando no hay proteína adsorbida en la superficie de las partículas, son las moléculas de surfactante las responsables de la reorganización interna del agregado (18). A altos grados de recubrimiento, el sistema se encuentra estabilizado estéricamente, observándose una cinética de agregación muy lenta que no conduce a la formación de estructuras fractales.

El radio hidrodinámico medio de los agregados, $\left\langle R_{h}\right\rangle$, se midió por dispersión dinámica de luz durante todo el proceso de agregación. A partir de aquí y considerando el tamaño de las partículas monoméricas, $\left\langle R_{0}\right\rangle$, se obtuvo el número medio de partículas por agregado, $\left\langle n>=\left(\left\langle R_{h}\right\rangle \mid<R_{0}>\right)^{-d f}\right.$. Para tiempos largos de agregación se observa un comportamiento asintótico en ley potencias, $<n>\propto t^{1 /(1-\lambda)}$ de donde se obtuvo el 
parámetro $\lambda$ (15). También por dispersión dinámica de luz, se obtuvo la dependencia temporal del primer cumulante de la función de autocorrelación de la intensidad dispersada, $\mu_{1}$. A partir de la ecuación [6] y una vez conocidos los valores de $d_{f}$ y $\lambda$, se calcularon las velocidades de reacción correspondientes a cada uno de los procesos de agregación estudiados (15). La figura 3 muestra las velocidades de reacción en función del grado de recubrimiento para $0.495 \mathrm{M}$ y $0.700 \mathrm{M}$ de $\mathrm{KCl}$, respectivamente. A altas concentraciones de electrolito se observa una curva en forma de campana con un máximo bien definido a coberturas superficiales intermedias. Esto indica, según el modelo de Healy y La Mer, que la floculación por puenteo podría ser el mecanismo de agregación predominante. Sin embargo, a bajas concentraciones de electrolito, la agregación es más lenta cuando aumenta el grado de recubrimiento, lo que sugiere que en este caso no existe floculación por puenteo. El modelo de Healy y La Mer no puede explicar ninguno de los comportamientos aquí mostrados, puesto que las constantes de velocidad obtenidas son un factor dos mayores que las predichas por este modelo. Los valores experimentales están muy cercanos al valor comúnmente aceptado para agregación controlada por difusión, que es de $6 \times 10^{-12} \mathrm{~cm}^{3} \mathrm{~s}^{-1}$, lo que indica que todas las colisiones en coagulación, puenteo y floculación débil deben ser efectivas.

Fijando los datos experimentales a la ecuación [2] podemos separar y cuantificar la aportación de cada mecanismo de agregación. Como condición de contorno, se identifica la constante de velocidad experimental para $\theta=0$ con la velocidad de reacción para el mecanismo de coagulación, $k_{c}$. El mejor ajuste experimental de la ecuacion [3] para $0.495 \mathrm{M}$ y $0.700 \mathrm{M}$ se muestra en la figura 3. Los valores obtenidos para las velocidades de reacción correspondientes a floculación débil, $k_{f d^{\prime}} \mathrm{y}$ a floculación por puenteo, $k_{f p}$, se muestran en la tabla I. Se encontró que, independientemente de la concentración electrolito, el mecanismo de floculación débil no se detecta, siendo predominante la floculación por puenteo en ambos casos. Esto significa que la probabilidad de choque y unión entre una parte recubierta de una partícula y una parte sin recubrir de otra partícula es más elevada que entre dos partes recubiertas, y del mismo orden de magnitud que entre dos partes sin recubrir. Este comportamiento se esperaba a altas concentraciones de electrolito, donde la forma de campana que mostraba la dependencia de $k_{\mathrm{s}}$ con el grado de recubrimiento, indicaba un claro predominio de la floculación por puenteo sobre los otros mecanismos de agregación. Sin embargo, el valor de $k_{f p}$ obtenido para $0.495 \mathrm{M}$ contradice la idea intuitiva formada al principio de que este mecanismo no estaba presente. A pH4.8 las moléculas de BSA están muy cercanas a su punto isoeléctrico y por tanto no alteran la carga neta de las partículas coloidales. Por esto no es sorprendente que las constantes de velocidad que caracterizan la floculación por puenteo sean - dentro de los errores experimentales - muy cercanas a las de coagulación.

\section{CONCLUSIONES}

Se estudió el efecto que las moléculas de proteína adsorbidas en la superficie de las microesferas de poliestireno tienen tanto en la estructura de los agregados como en el mecanismo de agregación. Para ello se determinó la dimensión fractal de los agregados, $d_{f}$ y las velocidades de reacción, $k_{s}$, como una función del recubrimiento superficial y para diferentes propiedades electroquímicas del medio de suspensión.

Las dimensiones fractales se midieron por SLS. Se observó que la dimensión fractal de los agregados aumenta con el grado de recubrimiento, lo que significa que la distribución
TABLA I. VelocidAdES DE AGREGACiÓN

\begin{tabular}{|c|c|c|c|}
\hline $\begin{array}{c}{[\mathrm{KCl}]} \\
\mathbf{M})\end{array}$ & $\begin{array}{c}\boldsymbol{k}_{c} \\
\left(\mathrm{~cm}^{3} \mathbf{s}^{-1}\right)\end{array}$ & $\begin{array}{c}\boldsymbol{k}_{f d} \\
\left(\mathrm{~cm}^{3} \mathbf{s}^{-\mathbf{1}}\right)\end{array}$ & $\begin{array}{c}\boldsymbol{k}_{f p} \\
\left(\mathrm{~cm}^{3} \mathbf{s}^{-\mathbf{1}}\right)\end{array}$ \\
\hline 0.495 & $(5.9 \pm 0.5) 10^{-12}$ & $(0.6 \pm 0.8) 10^{-12}$ & $(6.5 \pm 1.3) 10^{-12}$ \\
\hline 0.700 & $(6.0 \pm 0.4) 10^{-12}$ & $(0.6 \pm 0.9) 10^{-12}$ & $(7.6 \pm 1.5) 10^{-12}$ \\
\hline
\end{tabular}

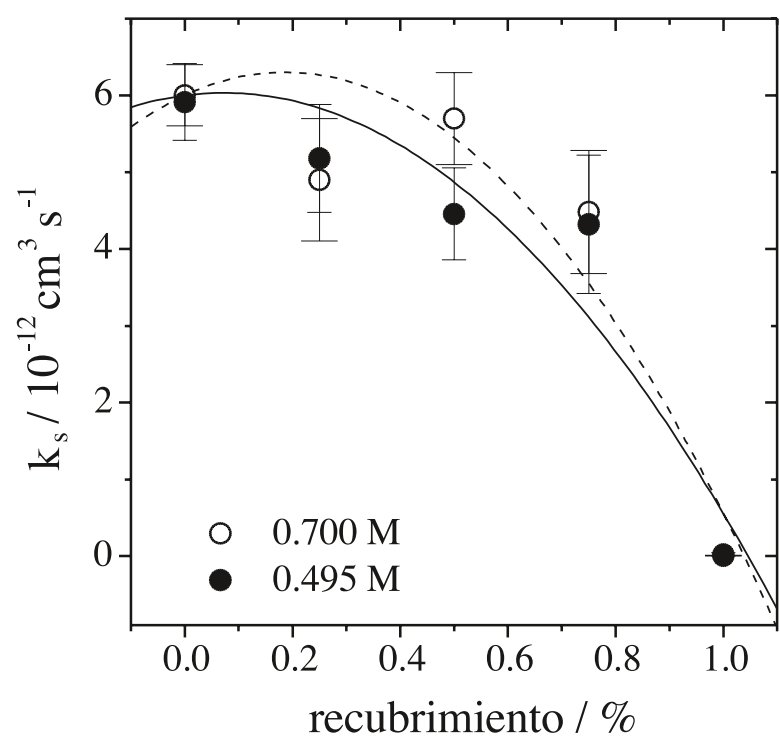

Figura 3: Dependencia de la velocidad de agregación con el grado de cobertura superficial a 0.495 M y 0.700 M de concentración iónica.

espacial de masa en el interior de los agregados depende de las características superficiales de los mismos. Los valores obtenidos indican la existencia de un proceso de reestructuración en el interior de los agregados, dando lugar a estructuras más compactas de las que se esperan para un proceso controlado únicamente por la difusión de los agregados.

Finalmente, se determinaron las velocidades de agregación, $k_{s}$, como una función de la cobertura superficial. Esto permitió separar y cuantificar las constantes de velocidad para los diferentes mecanismos de agregación (coagulación, floculación débil y floculación por puenteo) presentes en el proceso de agregación. Se observó que bajo las condiciones experimentales aquí estudiadas, los mecanismos de coagulación y floculación por puenteo están controlados por la difusión de los agregados mientras que la floculación débil no se detecta.

\section{AGRADECIMIENTOS}

Este trabajo ha sido financiado por la CICYT, proyecto MAT97-1024. Deseamos expresar nuestra gratitud a Manuel Quesada por habernos facilitado el sistema coloidal empleado en este trabajo.

\section{BIBLIOGRAFÍA}

1. E. Dickinson, L. Eriksson, "Particle flocculation by adsorbing polymers", Adv Colloid Interface Sci., 34, 1-29 (1991).

2. A. Fernández-Barbero, M. Cabrerizo, R. Martínez, R. Hidalgo-Alvarez, "Coagulation of polymer colloids by inmuno gamma globulin molecules", Prog. Colloid Polym. Sci., 93, 269-272, (1993).

3. T. W. Healy and V. K. La Mer, "The adsorption-flocculation reactions of a polymer with an aqueous colloidal dispersion", J. Phys. Chem., 66, 1835-

4. R.1838 (1962) 
Interface Sci., 102, 232-236, (1984).

5. S. G. Ash, E. J. Calyfield, "Effect of polymers on the stability of colloids", J. Colloid Interface Sci., 55, 645-657, (1976).

6. B. M. Moudgil, B. D. Shah, H. S. Soto, "Collision efficiency factors in polymer flocculation of fine particles", J. Colloid Interface Sci., 119, 466-473, (1987).

7. A. Molski, "On the collision efficiency approach to flocculation", Colloid Polym. Sci., 267, 371-375, (1989).

8. A. Schmitt, A. Fernández-Barbero, M. A. Cabrerizo-Vílchez, R. HidalgoAlvarez, "On the identification of bridging flocculation: An extended collision efficiency model", Prog. Colloid Polym. Sci., 110, 105-109, (1998).

9. M. V. Smoluchowski, "Versuch einer mathematischen theorie der koagulationskinetik kolloider löusungen", Z. Phys. Chem. 92, 129-168, (1917).

10. L. A. Spielman, "Viscous interactions in Brownian coagulation", J. Colloid Interface Sci., 33, 562-571, (1970).

11. H. Holthoff, A. Schmitt, A. Fernández-Barbero, M. Borkovec, M. A Cabrerizo-Vílchez, P. Schurtenberger, R. Hidalgo-Alvarez, “Measurement of Absolute Coagulation Rate Constants for Colloidal Particles: Comparison of Single and Multiparticle Light Scattering Techniques", J. Colloid Interface Sci., 192, 463-470, (1997).

12. R. Pecora, “Dynamic Light Scattering"; Pecora, R., Ed.; Plenum Press: New
York, (1985).

13. D. E. Koppel, "Analysis of macromolecular polydispersity in intensity correlation spectroscopy: The method of cumulants", J. Chem. Phys. 57, 4814-4820, (1972)

14. B. J. Olivier, C. M. Sorensen, "Evolution of the cluster size distribution during slow colloid aggregation", J. Colloid Interface Sci., 134, 139-146, (1990).

15. M. Tirado-Miranda, A. Schmitt, J. Callejas-Fernández, A. FernándezBarbero, "Dynamic scaling and fractal structure of small colloidal clusters", Colloids Surf. A, 162, 67-73, (2000).

16. M. Tirado-Miranda, A. Schmitt, J. Callejas-Fernández, A. FernándezBarbero, "Experimental study of fractal aggregation by static and dynamic light scattering", Prog. Colloid Polym. Sci., 104, 138-140, (1997)

17. J. A. Molina-Bolivar, "Mecanismos de estabilidad coloidal. Teoría y Aplicación a inmunoensayos", Tesis Doctoral, Universidad de Granada, (1999)

18. M. Tirado-Miranda, A. Schmitt, J. Callejas-Fernández, A. FernándezBarbero, "Colloidal Clusters with Finite Binding Energies: Fractal Structure and Growth Mechanism, Langmuir", 15(10), 3437-3444, (1999) 